Rev. Mat. Iberoamericana 23 (2007), no. 1, 1-16

\title{
Local Fatou theorem and the density of energy on manifolds of negative curvature
}

Frédéric Mouton

\begin{abstract}
Let $u$ be a harmonic function on a complete simply connected manifold $M$ whose sectional curvatures are bounded between two negative constants. It is proved here a pointwise criterion of nontangential convergence for points of the geometric boundary: the finiteness of the density of energy, which is the geometric analogue of the density of the area integral in the Euclidean half-space.
\end{abstract}

\section{Introduction}

If the study of non-tangential convergence of harmonic functions began in 1906 with the well-known theorem of Fatou (see [11]), it became clear in the 1970s (see for example [14]) that spaces of negative curvature provide a natural "geometric" setting for this study: as can be seen in section 1, several notions have simpler or more natural expressions in this geometric setting. From this point of view, we proved some years ago two pointwise criteria of non-tangential convergence -non-tangential boundedness and finiteness of the non-tangential energy - on Riemannian manifolds of pinched negative curvature by the use of Brownian motion (see [16]). We refer the reader to this article for the historical details and references on the study of nontangential convergence. Many questions arised as a consequence of these results. One was the existence of discrete analogues and we solved recently the case of trees (see [17]). Another was to find a geometric criterion corresponding to the Euclidean one on the density of the area integral given by J. Brossard (see [6]) and the purpose of this article is to give an answer.

2000 Mathematics Subject Classification: 31C12, 31C35, 58J65, 60J45.

Keywords: Harmonic functions, Fatou type theorems, area integral, negative curvature, Brownian motion. 
In section 1, we recall that the geometric interpretation of the area integral is the non-tangential energy. In section 4, we introduce the density of energy as a geometric interpretation of the density of the area integral. Again, this geometric expression is simpler than the Euclidean one. In order to prove that the finiteness of this density implies almost everywhere the non-tangential convergence we first recall a local Fatou theorem due to Hitoshi Arai (see [4]) in section 3. A new proof of that result is also given by refining arguments used in a previous work (see [16]). A straightforward corollary of this local Fatou theorem is a pointwise one: non-tangential boundedness from below is almost everywhere equivalent to non-tangential convergence. We then prove the main result in section 4 by a geometric interpretation of the proof of J. Brossard (see [6]), using for that some of our previous estimates. The geometric notions used along this article are introduced in section 1 and the probabilistic ones in section 2. This will be done briefly since the reader can refer to the previous article for more details (see [16]).

\section{A geometric setting}

The idea of studying non-tangential convergence on spaces of negative curvature came from the following observation: if you consider the Euclidean half-space with the standard hyperbolic metric, several non-tangential notions have more natural expressions. The boundary becomes an ideal one -it is at infinity-, the rays become geodesic rays, non-tangential cones become tubular neighbourhoods of geodesic rays and the area integral becomes an energy -an integral of a gradient to the square. We will now introduce these notions in the more general setting of pinched negative curvature.

Let $M$ be a complete simply connected Riemannian manifold whose sectional curvatures are bounded between two negative constants:

$$
-b^{2} \leq K \leq-a^{2}<0
$$

It has been known since Hadamard and Cartan that $M$ is diffeomorphic to the open ball $B^{d}$ with $d=\operatorname{dim} M$. Denote by $\Delta$ the Laplace-Beltrami operator on $M$ and by $G$ the associated Green function. As shown by M.T. Anderson and R. Schoen (see [3]) and also A. Ancona (see [1]), the Martin boundary of $M$, defined by the Green kernels, and its geometric boundary, defined by the geodesic rays, agree. We will denote this unique boundary by $\partial M$.

There are natural measures on $\partial M$ when dealing with harmonic functions and Brownian motion: the harmonic measures $\mu_{x}, x \in M$, which can be 
defined either as exit laws of Brownian motion starting at $x$ or by solving the Dirichlet problem at infinity (see [19]). We thus obtain a family $\mu=$ $\left(\mu_{x}\right)_{x \in M}$ of equivalent measures and then a notion of $\mu$-negligibility. Fixing a basepoint $o \in M$, and defining the Poisson Kernel $p_{\theta}(x)$ as limit of the Green kernels (normalised at $o$ ), we also have

$$
p_{\theta}(x)=\left(d \mu_{x} / d \mu_{o}\right)(\theta)
$$

(the Radon-Nykodim derivative).

Now let us define the non-tangential notions. Let $\gamma_{\theta}$ be the geodesic ray from $o$ to $\theta \in \partial M$. If $c>0$ and $\theta \in \partial M$, denote by

$$
\Gamma_{c}^{\theta}=\left\{x \in M \mid d\left(x, \gamma_{\theta}\right)<c\right\}
$$

the (non-tangential) tube of radius $c$ at $\theta$. A sequence of points of $M$ converges non-tangentially to $\theta$ if it converges to $\theta$ staying in a tube at $\theta$. A function $u$ converges non-tangentially at $\theta$ if, for all $c>0, u(x)$ has a limit as $x$ tends to $\theta$ in $\Gamma_{c}^{\theta}$. The function $u$ is said to be non-tangentially bounded at $\theta$ if it is bounded on each $\Gamma_{c}^{\theta}, c>0$. The non-tangential energy of $u$ on $\Gamma_{c}^{\theta}$ is the quantity

$$
J_{c}^{\theta}(u)=\int_{\Gamma_{c}^{\theta}}|\nabla u(x)|^{2} v_{M}(d x)=\|\nabla u\|_{L^{2}\left(\Gamma_{c}^{\theta}\right)}^{2},
$$

where $v_{M}$ denotes the Riemannian volume on $M$. The function $u$ is said to have a finite non-tangential energy at $\theta$ if

$$
J_{c}^{\theta}(u)<+\infty \quad \text { for all } c>0
$$

The main result of our previous work (see [16]) is the following:

Theorem 1 Let $M$ be a complete simply connected manifold of pinched negative curvature and $u$ be a harmonic function on $M$. Then, for $\mu$-almost all $\theta \in \partial M$, the following properties are equivalent:

(i) The function $u$ converges non-tangentially at $\theta$.

(ii) The function $u$ is non-tangentially bounded at $\theta$.

(iii) The function $u$ has a finite non-tangential energy at $\theta$.

The proof uses asymptotic Brownian notions as does the present work. They will be introduced in the next section. 


\section{Brownian motion and conditioning}

Let us denote by $\left(X_{t}(\omega)\right)_{t}$ the Brownian motion on $M$ which is defined as the diffusion associated to the Laplace-Beltrami operator. It is known under our hypotheses on $M$ that there is no explosion, i.e. the process is defined for all $t \geq 0$, and that Brownian motion converges almost surely to a point of $\partial M$ (see [18]). It is possible to chose as probability space the canonical space $\Omega=\mathcal{C}\left(\mathbb{R}_{+}, M\right)$ which is the space of continuous paths defined for $t \geq 0$. Then $X_{t}(\omega)=\omega(t)$ and the law of Brownian motion is given by probabilities $P_{x}, x \in M$ which are the probabilities obtained when Brownian motion starts at $x$. The above result can be now formulated as follows: for $P_{x}$-almost all $\omega \in \Omega$, there exists $\theta \in \partial M$ such that

$$
\lim _{t \rightarrow+\infty} X_{t}(\omega) \quad\left(=\lim _{t \rightarrow+\infty} \omega(t)\right)=\theta .
$$

Denoting this limit by $X_{\infty}(\omega)$, the image law of $P_{x}$ by $X_{\infty}$ is the harmonic measure $\mu_{x}$ as claimed in the last section.

By Doob's $h$-processes method, it is possible to condition Brownian motion to exit at a fixed point $\theta \in \partial M$ (see [10]). We thus obtain a probability $P_{x}^{\theta}$ on $\Omega$ that verifies a strong Markov property and an asymptotic zero-one law (see [16]). We can reconstruct the probability $P_{x}$ with these conditioned probabilities: for a suitable random variable $F$,

$$
E_{x}[F]=\int_{\partial M} E_{x}^{\theta}[F] \mu_{x}(d \theta) .
$$

A fundamental formula is the following: if $T$ is an almost surely finite stopping time and $\mathcal{F}_{T}$ is the associated $\sigma$-algebra, then for a non-negative $\mathcal{F}_{T^{-}}$ measurable function $F$,

$$
E_{x}^{\theta}[F]=\frac{1}{p_{\theta}(x)} E_{x}\left[F \cdot p_{\theta}\left(X_{T}\right)\right]
$$

The asymptotic zero-one law implies that for a function $u$ on $M$ and a given point $\theta \in \partial M$, the quantity $u\left(X_{t}\right)$ has a finite limit $(t \rightarrow+\infty)$ with $P_{x}^{\theta}$-probability 0 or 1 . In the second case we say that there is Brownian convergence of $u$ at the point $\theta$. This definition does not depend on the point $x$ and in the case of Brownian convergence the value of the limit depends on neither $\omega$ nor $x$. It is possible to define in the same way notions of Brownian boundedness and finiteness of the Brownian energy and it is a straightforward consequence of martingale theorems that these three notions are $\mu$-almost everywhere equivalent when $u$ is harmonic. However they are not equivalent to the corresponding non-tangential notions, which are stronger. 


\section{Local and pointwise Fatou theorems}

We recall in this section a theorem which will be used for the proof of the criterion of the density of energy in section 4 . We begin with a definition. If $U$ is an open subset of $M$ and $\theta$ a point of $\partial M, \theta$ is said to be tangential for $U$ if the set $\Gamma_{c}^{\theta} \backslash U$ is bounded for each $c>0$.

The following result for admissible convergence in the sense of [4] is due to Hitoshi Arai (see [4]), who proved it by potential theoretic arguments. Later Patricio Cifuentes and Adam Korányi proved that admissible regions and non-tangential regions are equivalent (see [9]). Therefore as pointed out in [5], his result holds true for non-tangential convergence. We give here a different proof by the use of Brownian motion.

Theorem 2 ("Local Fatou theorem", H. Arai) Let $U$ be an open subset of $M$. If $u$ is a non-negative harmonic function on $U$ then for $\mu$-almost all $\theta$ that is tangential for $U$, the function $u$ converges non-tangentially at $\theta$.

To prove this theorem we will use two lemmas which generalise previous results (see [16]) and a proposition first proved by Alano Ancona in a potential theoretic setting (see [2]). A geometric-probabilistic proof of this proposition is given in the appendix.

Lemma 1 Let $U$ be an open subset of $M$. Then for $\mu$-almost all $\theta$ that are tangential for $U$, Brownian motion "ends its life" in $U P_{o}^{\theta}$-almost surely (i.e. $X_{t} \in U$ for $t$ big enough).

Lemma 2 Let $U$ be a connected open subset of $M$ such that $o \in U$ and let $\tau$ be the time for which Brownian motion exits $U$. Then for $\mu$-almost all $\theta$ that are tangential for $U, P_{o}^{\theta}[\tau=+\infty]>0$.

Proposition 1 (A. Ancona) Consider a sequence of balls of fixed (positive) radius whose centers converge non-tangentially to a point $\theta \in \partial M$.

Then Brownian motion meets $P_{o}^{\theta}$-almost surely an infinity of these balls.

Proof of Lemma 1. Let $c$ be a fixed positive real. Let $A$ be the set of tangential points for $U$ and, for $N \in \mathbb{N}$, let

$$
A_{N}=\left\{\theta \in \partial M \mid \Gamma_{c}^{\theta} \backslash U \subset B(o, N)\right\} .
$$

As $A$ is the countable union of the $A_{N}$, it is sufficient to prove the required property for $\mu$-almost all $\theta \in A_{N}$, for each $N \in \mathbb{N}$. Let $N$ be a fixed integer. It is known (see [16], corollaire 4.1) that for $\mu$-almost all $\theta \in A_{N}$, Brownian motion ends its life in $\Gamma_{c}\left(A_{N}\right)=\bigcup_{\theta^{\prime} \in A_{N}} \Gamma_{c}^{\theta^{\prime}} P_{o}^{\theta}$-almost surely. Let $\theta$ be such 
a point. As Brownian motion goes to infinity $P_{o}^{\theta}$-almost surely, it leaves the ball $B(o, N)$ and then ends its life in $\Gamma_{c}\left(A_{N}\right) \backslash B(o, N)$ which is a subset of $U$. The property is then true for $\mu$-almost all $\theta \in A_{N}$ and lemma 1 is proved.

Proof of Lemma 2. The proof is similar to one in our previous article (see $[16$, p. 496]) and uses the maximum principle, lemma 1 and the strong Markov property. Let us recall the sketch: the function $h(x)=$ $p_{\theta}(x) P_{x}^{\theta}[\tau=+\infty]$ is non-negative harmonic and therefore either identically zero or positive at each point by the maximum principle on the connected open set $U$. Let $\tau_{N}$ denote the exit time of the ball $B(o, N)$. Then there exists $N$ such that

$$
P_{o}^{\theta}\left[" X_{t} \text { stays in } U \text { after } \tau_{N} "\right]>0,
$$

because the monotonous union of these events is almost sure by lemma 1 . Applying the strong Markov property at the stopping time $\tau_{N}$ gives the positivity of $h$ and the proof is completed.

We can now prove the theorem.

Proof of Theorem 2. As $U$ is open, it has a countable number of connected components and we can assume without loss of generality that $U$ is connected. We can also assume that $o \in U$ (note that the empty set has no tangential points). Denote by $\tau$ the exit time of the set $U$. It is well-known that $\left(u\left(X_{t}\right)\right)$ is a local martingale on $[0, \tau)$ since $u$ is harmonic. As $u$ is nonnegative, the local martingales convergence theorem tells us that the local martingale converges $P_{o}$-almost surely. Then by formula 2.1 , for $\mu$-almost all $\theta \in \partial M, u\left(X_{t}\right)$ converges $P_{o}^{\theta}$-almost surely. Combining this with lemma 2 gives that, for $\mu$-almost all $\theta$ that are tangential for $U$,

$$
P_{o}^{\theta}\left[" \tau=+\infty \text { and } u\left(X_{t}\right) \text { converges"] }>0 .\right.
$$

Let $\theta$ be such a point. Consider the function $\widetilde{u}$ defined on the whole manifold $M$ by $\widetilde{u}(x)=u(x)$ for $x \in U$ and $u(x)=0$ otherwise. As the above probability is positive, $\widetilde{u}\left(X_{t}\right)$ converges with positive $P_{o}^{\theta}$-probability as $t$ tends to $+\infty$ and therefore $P_{o}^{\theta}$-almost surely by the asymptotic zero-one law (section 2): we therefore have Brownian convergence of $\widetilde{u}$ at $\theta$. Taking the logarithm, we have Brownian convergence of $f=\ln (\widetilde{u}+1)$ at $\theta$. Let $l$ denote the corresponding finite limit.

Let us fix $c>0$. Since the function $u+1$ is harmonic and positive on $U$, the Cheng-Yau infinitesimal version of the Harnack inequality (see [8]) implies that $\ln (u+1)$ and therefore $f$ are uniformly continuous on $\Gamma=\Gamma_{c+1}^{\theta} \backslash$ $B(o, R)$, provided $R$ is chosen such that there exists an $\eta$-neighbourhood $(\eta>0)$ of $\Gamma$ contained in $U$, which is possible because $\theta$ is tangential. 
As shown in [16], this uniform continuity along with the Brownian convergence above insures that the limit of $f(x)$ at $\theta$ in $\Gamma_{c}^{\theta}$ exists and is $l$. Otherwise, there would exist $\epsilon>0$ and a sequence $\left(x_{n}\right)$ in $\Gamma_{c}^{\theta}$ such that $\lim _{n} x_{n}=\theta$ and $\left|f\left(x_{n}\right)-l\right| \geq \epsilon$ for all $n$. Without loss of generality, $x_{n}$ can be taken to lie outside $B(o, R+1)$ and therefore $B\left(x_{n}, 1\right) \subset \Gamma$. By uniform continuity of $f$ on $\Gamma$, there exists $0<\alpha<1$ such that for all $x, y \in \Gamma,|f(x)-f(y)| \leq \frac{\epsilon}{3}$ as soon as $d(x, y) \leq \alpha$. As $x_{n}$ goes non-tangentially to $\theta$, the Brownian motion meets $P_{o}^{\theta}$-almost surely an infinity of $B_{n}=B\left(x_{n}, \alpha\right)$ by proposition 1 . Let now $\left(X_{t}(\omega)\right)$ be a $P_{o}^{\theta}$-generic Brownian path: $\lim _{t \rightarrow+\infty} f\left(X_{t}(\omega)\right)=l$ and $\left(X_{t}(\omega)\right)$ meets an infinity of balls $B_{n}$. We can then choose a $t_{0}$ such that $\left|f\left(X_{t}(\omega)\right)-l\right| \leq \frac{\epsilon}{3}$ as soon as $t>t_{o}$. By compactness, truncated path $\left(X_{t}(\omega)\right)_{t \leq t_{O}}$ meets only a finite number of $B_{n}$ and the path $\left(X_{t}(\omega)\right)_{t>t_{0}}$ therefore meets at least one ball $B_{n_{1}}$. We can then choose $t_{1}>t_{0}$ such that $X_{t_{1}}(\omega) \in B_{n_{1}}$, for which

$$
0<\epsilon \leq\left|f\left(x_{n_{1}}\right)-l\right| \leq\left|f\left(x_{n_{1}}\right)-f\left(X_{t_{1}}(\omega)\right)\right|+\left|f\left(X_{t_{1}}(\omega)\right)-l\right| \leq \frac{2 \epsilon}{3},
$$

a contradiction which proves that the limit of $f(x)$ at $\theta$ in $\Gamma_{c}^{\theta}$ is $l$.

As this is true for any $c>0$, on taking the exponential we see that the function $u$ is non-tangentially convergent at $\theta$. Since this is true for $\mu$-almost all tangential $\theta$, the theorem is proved.

A consequence of this theorem is that non-tangential boundedness from below (or above) is a pointwise criterion of non-tangential convergence, which corresponds to a result of L. Carleson in the Euclidean case (see [7]):

Corollary 1 ("Pointwise Fatou theorem", H. Arai) Let $M$ be a complete simply connected manifold of pinched negative curvature and let $u$ be a harmonic function on $M$. Then, for $\mu$-almost all $\theta \in \partial M$, the following properties are equivalent:

(i) The function $u$ converges non-tangentially at $\theta$.

(ii) For all $c>0, u$ is bounded from below on $\Gamma_{c}^{\theta}$.

(iii) There exists $c>0$ such that $u$ is bounded from below on $\Gamma_{c}^{\theta}$.

Proof of Corollary 1. The only thing to prove is that (iii) implies (i). Fix $c>0$ and, for $N \in \mathbb{N}$, let

$$
A_{N}=\left\{\theta \in \partial M \mid \forall x \in \Gamma_{c}^{\theta}, u(x) \geq-N\right\} .
$$

By countable union, it is sufficient to prove that $u$ converges non-tangentially at $\mu$-almost all points of $A_{N}$, for each $N \in \mathbb{N}$. Let $N$ be a fixed integer. Take

$$
U=\bigcup_{\theta \in A_{N}} \Gamma_{c}^{\theta} .
$$


The function $u+N$ is non-negative harmonic on $U$ and by theorem $2, u+N$ is non-tangentially convergent at $\mu$-almost all tangential points for $U$, and therefore so is the function $u$. It is known (see [16], corollaire 4.2) that $\mu$-almost all $\theta \in A_{N}$ are tangential for $U$, which completes the proof.

\section{The density of the energy}

The density of the area integral for harmonic functions on the Euclidean half-space has been introduced in 1983 by R.F. Gundy (see [13] and [12]). It is defined by the formulas:

$$
D^{r}(x)=\frac{1}{2} \int_{\Gamma(x)} y^{1-\nu} \Delta|u-r|(d z)=\int_{\Gamma(x)} y^{1-\nu}|\nabla u(z)| \sigma_{r}(d z),
$$

where points of half-space $\mathbb{R}^{\nu} \times(0,+\infty)$ are denoted by $z=(x, y), \Gamma(x)$ is a non-tangential cone over $x$ and $\sigma_{r}$ is the hypersurface measure on the level set $\{u=r\}$.

Formulating these expressions with the hyperbolic metric, the term $y^{1-\nu}$ disappears, which leads us to introduce in our geometric setting, for $u$ harmonic, $\theta \in \partial M$ and $c>0$ :

$$
D_{c}^{r}(\theta)=-\frac{1}{2} \int_{\Gamma_{c}^{\theta}} \Delta|u-r|(d x)
$$

and

$$
\widetilde{D}_{c}^{r}(\theta)=\int_{\Gamma_{c}^{\theta}}|\nabla u(x)| \sigma_{r}(d x),
$$

where $\sigma_{r}$ is the hypersurface measure on $\{u=r\}$. The minus sign is due to the geometers' convention for the sign of $\Delta$. As in the Euclidean case, the positive measures $-(1 / 2) \Delta|u-r|$ (recall that $|u-r|$ is subharmonic) and $|\nabla u| \sigma_{r}$ are equal as distributions outside singular points of $u$, and $D_{c}^{r}(\theta)=$ $\widetilde{D}_{c}^{r}(\theta)$ for almost all $r \in \mathbb{R}$ by Sard's theorem. J. Brossard has shown the equality for all $r$ in the Euclidean case (see [6]). We will use only the first expression, which will be called the density of the energy, but it is the second which has given the name of "density": the geometric "co-area" formula gives, for a non-negative function $f$ on $\mathbb{R}$, the equation

$$
\int_{\Gamma_{c}^{\theta}} f(u(x))|\nabla u(x)|^{2} v_{M}(d x)=\int_{\mathbb{R}} f(r)\left(\int_{\Gamma_{c}^{\theta}}|\nabla u(x)| \sigma_{r}(d x)\right) d r .
$$

Our goal is here to prove the following criterion of non-tangential convergence: 
Theorem 3 Let $M$ be a complete simply connected manifold of pinched negative curvature, $u$ a harmonic function on $M$ and $c>0$.

Then $u$ is non-tangentially convergent at $\mu$-almost all $\theta$ such that $D_{c}^{0}(\theta)$ is finite.

Proof of Theorem 3. As before, it is sufficient to prove for all $N \in \mathbb{N}$ that $u$ is non-tangentially convergent at $\mu$-almost all points of the set

$$
A_{N}=\left\{\theta \in \partial M \mid D_{c}^{0}(\theta) \leq N\right\}
$$

By integration on $A_{N}$, we obtain

$$
N \geq \int_{A_{N}} D_{c}^{0}(\theta) \mu_{o}(d \theta)=-\frac{1}{2} \int_{A_{N}}\left(\int_{\Gamma_{c}^{\theta}} \Delta|u|(d x)\right) \mu_{o}(d \theta)
$$

which, with the notation $\Gamma=\bigcup_{\theta \in A_{N}} \Gamma_{c}^{\theta}$ and $H_{c}(x)=\left\{\theta \in \partial M \mid x \in \Gamma_{c}^{\theta}\right\}$, is equal to

$$
-\frac{1}{2} \int_{\Gamma} \mu_{o}\left(H_{c}(x) \cap A_{N}\right) \Delta|u|(d x) .
$$

This integral is finite and the idea is to "replace" the term $\mu_{o}\left(H_{c}(x) \cap A_{N}\right)$ by a Green kernel, which will enable a Brownian interpretation. For that we use an estimate that can be found in our previous article (see [16], p. 499), which relies on the Harnack principle at infinity: if $u_{A_{N}}$ is the bounded harmonic function defined by $u_{A_{N}}(x)=P_{x}\left[X_{\infty} \in A_{N}\right]$ then there exists $\alpha \in(0,1)$ and $C, c^{\prime}>0$ such that $\left\{u_{A_{N}}>\alpha\right\} \subset \Gamma$ and

$$
\forall x \in\left\{u_{A_{N}}>\alpha\right\} \backslash B\left(o, c^{\prime}\right), \quad G(o, x) \leq C \cdot \mu_{o}\left(H_{c}(x) \cap A_{N}\right) .
$$

For convenience, we will denote $V=\left\{u_{A_{N}}>\alpha\right\}$. As a consequence of the above estimates,

$$
-\int_{V \backslash B\left(o, c^{\prime}\right)} G(o, x) \Delta|u|(d x)<+\infty .
$$

We need now the following lemma, which will be proved later:

Lemma 3 Let $U$ be a bounded regular domain of $M$ such that $o \in U$, let $G_{U}$ be the associated Green function, let $\tau$ be the exit time of $U$ and let $u$ be a harmonic function on $M$. Then

$$
E_{o}\left[\left|u\left(X_{\tau}\right)\right|\right]=|u(o)|-\int_{U} G_{U}(o, x) \Delta|u|(d x) .
$$


Applying this lemma with $U=B\left(o, c^{\prime}\right)$ gives

$$
-\int_{B\left(o, c^{\prime}\right)} G_{B\left(o, c^{\prime}\right)}(o, x) \Delta|u|(d x)<+\infty .
$$

On the other hand, the function $f$ defined by

$$
f(x)=G(o, x)-G_{B\left(o, c^{\prime}\right)}(o, x)
$$

is positive harmonic on $B\left(o, c^{\prime}\right)$ and can be extended continuously to $\bar{B}\left(o, c^{\prime}\right)$. Since $-\Delta|u|$ is a positive measure which is finite on compact sets,

$$
-\int_{B\left(o, c^{\prime}\right)} f(x) \Delta|u|(d x) \leq-\int_{\bar{B}\left(o, c^{\prime}\right)} f(x) \Delta|u|(d x)<+\infty
$$

so

$$
-\int_{B\left(o, c^{\prime}\right)} G(o, x) \Delta|u|(d x)<+\infty
$$

and

$$
I=-\int_{V} G(o, x) \Delta|u|(d x)<+\infty .
$$

Applying lemma 3 for an increasing sequence of compact regular domains $V_{n}$ such that $\bigcup_{n} V_{n}=V$, and using the fact that $G_{V_{n}} \leq G$, gives for all $n$ :

$$
E_{o}\left[\left|u\left(X_{\tau_{n}}\right)\right|\right] \leq|u(o)|+I,
$$

where $\tau_{n}$ is the exit time of $V_{n}$. So $\sup _{n} E_{o}\left[\left|u\left(X_{\tau_{n}}\right)\right|\right]$ is finite and a wellknown potential theoretic method (see [6], p. 307) gives a decomposition of $u$ as the difference of two non-negative harmonic functions on $V$. By the local Fatou theorem (theorem 2), both converge non-tangentially at $\mu$-almost all tangential $\theta$ for $V$, and therefore so does $u$. The classical convergence result for bounded harmonic functions (see [16], théorème 4.1) tells us that $u_{A_{N}}$ converges non-tangentially to 1 at $\mu$-almost all $\theta \in A_{N}$. Such a $\theta$ is then tangential for $V$ by definition of $V$. So $u$ converges non-tangentially at $\mu$-almost all $\theta$ of $A_{N}$ and the proof is completed.

The proof of the lemma remains:

Proof of Lemma 3. Using the Green formula twice, as $G_{U}(o, \cdot)$ vanishes on $\partial U$,

$$
\begin{aligned}
\int_{U} G_{U}(o, x) \Delta|u|(d x)=\int_{U} \Delta_{x} G_{U}(o, x)|u(x)| v_{M}(d x) \\
\quad-\int_{\partial U} \frac{\partial_{x} G_{U}(o, x)}{\partial \vec{N}}|u(x)| v_{M}(d x)
\end{aligned}
$$

where $\vec{N}$ is the interior normal to $\partial U$. 
By definition of the Green function,

$$
\int_{U} \Delta_{x} G_{U}(o, x)|u(x)| v_{M}(d x)=|u(o)|
$$

and it is known that $\frac{\partial_{x} G_{U}(o, x)}{\partial \vec{N}} v_{M}$ is equal to the harmonic measure of $U$ starting at $o$. Then

$$
\int_{U} G_{U}(o, x) \Delta|u|(d x)=|u(o)|-\int_{\partial U}|u(x)| \mu_{o}^{U}(d x)=|u(o)|-E_{o}\left[\left|u\left(X_{\tau}\right)\right|\right] .
$$

\section{Appendix}

Here we give a geometric-probabilistic proof of Ancona's result (proposition 1):

For a sequence of balls of fixed positive radius whose centers converge non-tangentially to $\theta \in \partial M$, Brownian motion meets $P_{o}^{\theta}$-almost surely an infinity of balls.

We will need the two following Harnack type propositions. The first one is the usual Harnack property for balls. It is a direct consequence of Cheng-Yau infinitesimal Harnack inequality (see [8]).

Proposition 2 ("Harnack property for balls") Let $r$ and $R$ be two radii such that $0<r<R$. There exists a constant $C_{1}$ depending only on dimension of $M$ and the lower bound of the curvature such that, for all $x \in M$ and all positive harmonic function $u$ on $B(x, R)$, we have

$$
\sup _{z \in \bar{B}(x, r)} u(z) \leq C_{1} \cdot \inf _{z \in \bar{B}(x, r)} u(z) .
$$

The second one is the Harnack principle at infinity, which is the key step used in the identification of geometric and Martin boundaries (see [3], [1]).

We introduce some notations. For $x \in M$, a tangent vector $\xi \in T_{x} M$ and an angle $\alpha$, we denote by $\Lambda(x, \xi, \alpha)$ the open cone of vertex $x$, direction $\xi$ and angle $\alpha$ i.e. the union of all (open) geodesic rays starting at $x$ and whose angles with respect to $\xi$ are smaller than $\alpha$. We denote by

$$
\bar{\Lambda}(x, \xi, \alpha)
$$

the corresponding closed cone (not the same as the closure $\overline{\Lambda(x, \xi, \alpha)}$ of the cone in $\bar{M}$ ) and by

$$
T(x, \xi, \alpha)=\bar{\Lambda}(x, \xi, \alpha) \backslash B(x, 1)
$$

the corresponding truncated closed cone. 
Theorem 4 ("Harnack principle at infinity") Let $\alpha \in(0, \pi / 2)$ be an acute angle. There exists a constant $C_{2}$ depending only on the dimension of $M$ and curvature bounds such that for all $x \in M$ and $\xi \in T_{x} M$ we have the following property:

If $u$ and $v$ are two positive harmonic functions on $\Lambda(x, \xi, \alpha)$, having limit 0 at infinity (for the topology of $\bar{M}$ ), then

$$
\sup _{z \in T} \frac{u(z)}{v(z)} \leq C_{2} \cdot \inf _{z \in T} \frac{u(z)}{v(z)}
$$

where $T=T(x, \xi, \alpha / 2)$.

Using these results, we will first prove the following technical lemma which invites comparison with Ancona's probabilistic interpretation of the Harnack principle at infinity (see [1]):

Lemma 4 Given an acute angle $\alpha \in(0, \pi / 2)$ and a radius $R>1$, there exists a constant $C_{3}$ depending only on the dimension of $M$ and curvature bounds such that:

If a compact $K$ of $M, y, z \in M, \xi \in T_{y} M$ and $\theta \in \partial M$ verify the four following properties

- $K \cap \Lambda(y, \xi, \alpha)=\emptyset$

- $K \subset \bar{B}(y, R)$

- $z \in T(y, \xi, \alpha / 2)$

- $\theta \notin \overline{\Lambda(y, \xi, \alpha)}$

then, for all $x \in T(y, \xi, \alpha / 2) \cap \bar{B}(y, R)$,

$$
P_{z}^{\theta}\left[\text { "X meets } K \text { "] } \geq C_{3} \cdot P_{x}\left[\text { " } X_{t} \text { meets } K\right. \text { "]. }\right.
$$

Proof of Lemma 4. Let $C_{1}$ be the constant obtained using the Harnack property for balls with radii $R$ and $2 R$, and $C_{2}$ the constant obtained using the Harnack principle at infinity with angle $\alpha$. These constants depend only on dimension of $M$ and curvature bounds.

Let $x \in T(y, \xi, \alpha / 2) \cap \bar{B}(y, R)$. The function $f: w \mapsto P_{w}\left[\right.$ [ $X_{t}$ meets $K$ "] is known to be harmonic (using the strong Markov property for example). Let $\tau$ be the exit time of $K^{c}$, which is $+\infty$ if the trajectory doesn't meet $K$. By definition of the conditional probability $P_{z}^{\theta}$,

$$
P_{z}^{\theta}\left[\text { [X } X_{t} \text { meets } K "\right]=E_{z}^{\theta}\left[\mathbf{1}_{K}\left(X_{\tau}\right)\right]=\frac{1}{p_{\theta}(z)} E_{z}\left[\mathbf{1}_{K}\left(X_{\tau}\right) p_{\theta}\left(X_{\tau}\right)\right] .
$$


In the case of $\mathbf{1}_{K}\left(X_{\tau}\right) \neq 0, X_{\tau} \in K$, so $x$ and $X_{\tau}$ are both in $\bar{B}(y, R)$. As $p_{\theta}$ is positive harmonic on $B(y, 2 R)$ (since it is so on $M$ ), the Harnack property for balls gives us

$$
P_{z}^{\theta}\left[\text { "X } X_{t} \text { meets } K "\right] \geq C_{1} \cdot \frac{p_{\theta}(x)}{p_{\theta}(z)} \cdot f(z)=C_{1} \cdot f(x)\left(\frac{p_{\theta}(x)}{f(x)}\right)\left(\frac{f(z)}{p_{\theta}(z)}\right) .
$$

The functions $p_{\theta}$ and $f$ are harmonic on the cone $\Lambda(y, \xi, \alpha)$ and their restrictions on the cone tend to 0 at infinity (since $\theta \notin \overline{\Lambda(y, \xi, \alpha)}$ ). As $x$ and $z$ are in $T(y, \xi, \alpha / 2)$, the Harnack principle gives us

$$
\left(\frac{p_{\theta}(x)}{f(x)}\right)\left(\frac{f(z)}{p_{\theta}(z)}\right) \geq C_{2}
$$

which completes the proof of lemma 4.

A consequence of that lemma is the following:

Corollary 2 Fix $z \in M$ and $\theta \in \partial M$. Let $\left(K_{n}\right)$ be a sequence of compact sets of $M$ such that there exist an angle $\alpha \in(0, \pi / 2)$, a radius $R>1$ and a sequence $\left(y_{n}, \xi_{n}\right)$ in TM for which the hypotheses of lemma 4 are satisfied for all $n$.

Let $\left(x_{n}\right)$ be a sequence in $M$ such that $x_{n} \in T\left(y_{n}, \xi_{n}, \alpha / 2\right) \cap \bar{B}\left(y_{n}, R\right)$ for all $n$, and verifying

$$
\inf _{n} P_{x_{n}}\left[" X_{t} \text { meets } K_{n} "\right]>0
$$

Then we have

$$
P_{z}^{\theta}\left[\text { "X } X_{t} \text { meets an infinity of } K_{n} \text { "] }>0 .\right.
$$

Moreover, if for each ball $B, K_{n} \cap B=\emptyset$ for $n$ large enough, the above probability is 1 .

Proof of Corollary 2. Let $A_{n}$ be the event " $X_{t}$ meets $K_{n}$ ". As a direct consequence of lemma 4 ,

$$
\inf _{n} P_{z}^{\theta}\left[A_{n}\right]>0
$$

and

$$
P_{z}^{\theta}\left[\limsup _{n} A_{n}\right]=\inf _{n} P_{z}^{\theta}\left[\bigcup_{k \geq n} A_{k}\right] \geq \inf _{n} P_{z}^{\theta}\left[A_{n}\right]>0
$$

which proves the first assertion.

If the $K_{n}$ exit each ball, the event " $X_{t}$ meets an infinity of $K_{n}$ " is asymptotic (see [16]) and its probability is 1 by the zero-one law. 
Proof of Proposition 1. We can now prove Ancona's property. It can be supposed without loss of generality that the balls are closed (by taking closed balls of smaller radius). Let us denote by $B_{n}$ the balls and by $r$ their radius. It can also be supposed that $d\left(z, B_{n}\right) \geq 1$ for all $n$ since the centers converge to infinity. In order to apply the corollary above with $K_{n}=B_{n}$, we construct first the pairs $\left(y_{n}, \xi_{n}\right)$. Let $\gamma_{n}$ be the geodesic segment from $B_{n}$ 's center to z, $y_{n}$ be the intersection of $\gamma_{n}$ and $B_{n}$ 's boundary and $\xi_{n}$ be the vector of $T_{y_{n}} M$ pointing toward $z$.

It remains to choose the angle $\alpha$ and the radius $R$. Let $R=2 r+1$. The first, second and third hypothesis of lemma 4 are easily checked. The fourth will be verified for large $n$ by appropriate choice of $\alpha$.

The points $y_{n}$ converge non-tangentially to $\theta$, hence stay at a bounded distance $c$ of the geodesic ray $(z \theta)$. The angle $\widehat{y_{n} z \theta}$ tends to zero as $n$ goes to infinity and so is acute for $n$ large, which can be supposed true for all $n$ without loss of generality. It implies that the projection $t_{n}$ of $y_{n}$ onto the geodesic line defined by $z$ and $\theta$ lies between $z$ and $\theta$ i.e. on the ray $(z \theta)$. It can be shown (see [15]) by classical techniques using Toponogov's theorem that the angle $\widehat{z y_{n} \theta}$ is greater than or equal to the analogous angle of an ideal triangle of finite side length $d\left(y_{n}, z\right)$ and height $d\left(y_{n}, t_{n}\right)$ in the model of constant curvature $-b^{2}$. This analogue angle is itself greater than or equal to the angle $\beta$ of a right-angled ideal triangle of finite side length $c$ in the same model $\left(\right.$ as $\left.d\left(y_{n}, t_{n}\right) \leq c\right)$. We choose $\alpha=\beta / 2$ which implies $\widehat{z y_{n} \theta}>\alpha$ and hence $\theta \notin \overline{\Lambda\left(y_{n}, \xi_{n}, \alpha\right)}$.

To apply the corollary, it will be enough to choose $x_{n}$. Let $x_{n}$ be the point on the geodesic segment $\gamma_{n}$ at distance 1 from $y_{n}$ i.e. on the boundary of $T\left(y_{n}, \xi_{n}, \alpha / 2\right)$. Then

$$
P_{x_{n}}\left[\text { " } X_{t} \text { meets } B_{n} \text { "] } \geq P_{x_{n}}\left[\text { " } X_{t} \text { leaves } B\left(x_{n}, 1+r\right) \text { by } S\left(x_{n}, 1+r\right) \cap B_{n}\right. \text { "] }\right.
$$

The curvature being pinched, there exists $\gamma>0$ depending only upon $r$ and the curvature bounds, such that $B_{n} \cap S\left(x_{n}, 1+r\right)$ contains the intersection of $S\left(x_{n}, 1+r\right)$ and a cone of angle $\gamma$ and vertex $x_{n}$. By a known property of quasi-isotropy of harmonic measures (see [15]), the probability of leaving $B\left(x_{n}, 1+r\right)$ by that cone is more than a positive constant independent on $n$, which proves that

$$
\inf _{n} P_{x_{n}}\left[" X_{t} \text { meets } B_{n} "\right]>0 .
$$

As the $B_{n}$ exit from each fixed ball, applying the corollary finishes the proof.

Thanks. I am very grateful to Jean Brossard for all the discussions we have had and will have. 


\section{References}

[1] Ancona, A.: Negatively curved manifolds, elliptic operators and the Martin boundary. Ann. of Math. (2) 125 (1987), 495-536.

[2] Ancona, A.: Théorie du potentiel sur les graphes et les variétés. In École d'été de Probabilités de Saint-Flour XVIII-1988, 1-112. Lecture Notes in Math. 1427. Springer, Berlin, 1990.

[3] Anderson, M. T. And Schoen, R.: Positive harmonic functions on complete manifolds of negative curvature. Ann. of Math. (2) 121 (1985), 429-461.

[4] ArAi, H.: Boundary behavior of functions on complete manifolds of negative curvature. Tohoku Math. J. (2) 41 (1989), 307-319.

[5] Arai, H.: Hardy spaces, Carleson measures and a gradient estimate for the harmonic functions on negatively curved manifolds. In Taniguchi Conference on Mathematics Nara '98, 1-49. Adv. Stud. Pure Math. 31. Math. Soc. Japan, Tokyo, 2001.

[6] Brossard, J.: Densité de l'intégrale d'aire dans $\mathbb{R}_{+}^{n+1}$ et limites non tangentielles. Invent. Math. 93 (1988), 297-308.

[7] Carleson, L.: On the existence of boundary values for harmonic functions in several variables. Ark. Mat. 4 (1962), 393-399.

[8] Cheng, S.-Y. And YAu, S.-T.: Differential equations on Riemannian manifolds and their geometric applications. Comm. Pure Appl. Math. 28 (1975), 333-354.

[9] Cifuentes, P. and Korányi, A.: Admissible convergence in CartanHadamard manifolds. J. Geom. Anal. 11 (2001), 233-239.

[10] Doob, J.L.: Conditional brownian motion and the boundary limits of harmonic functions. Bull. Soc. Math. France 85 (1957), 431-458.

[11] Fatou, P.: Séries trigonométriques et séries de Taylor. Acta Math. 30 (1906), 335-400.

[12] Gundy, R.F. and Silverstein, M.L.: The density of the area integral in $\mathbb{R}_{+}^{n+1}$. Ann. Inst. Fourier (Grenoble) 35 (1985), 215-229.

[13] Gundy, R.F.: The density of the area integral. In Conference on harmonic analysis in honor of Antoni Zygmund, vol. I, II (Chicago, Ill., 1981), 138-149. Wadsworth Math. Ser.. Wadsworth, Belmont, CA, 1983.

[14] KorÁnyi, A. And Putz, R. B.: Local Fatou theorem and area theorem for symmetric spaces of rank one. Trans. Amer. Math. Soc. 224 (1976), $157-168$.

[15] Mouton, F.: Convergence non-tangentielle des fonctions harmoniques en courbure négative. PhD Thesis, Grenoble, 1994.

[16] Mouton, F.: Comportement asymptotique des fonctions harmoniques en courbure négative. Comment. Math. Helv. 70 (1995), 475-505. 
[17] Mouton, F.: Comportement asymptotique des fonctions harmoniques sur les arbres. In Séminaire de Probabilités XXXIV, 353-373. Lecture Notes in Math. 1729. Springer, Berlin, 2000.

[18] Prat, J.-J.: Étude asymptotique et convergence angulaire du mouvement brownien sur une variété à courbure négative. C. R. Acad. Sci. Paris Sér. A-B 280 (1975), A1539-A1542.

[19] Sullivan, D.: The Dirichlet problem at infinity for a negatively curved manifold. J. Differential Geom. 18 (1983), 723-732.

Recibido: 14 de mayo de 2003

Revisado: 18 de septiembre de 2006

Frédéric Mouton

Institut Fourier Université Grenoble 1

BP 74

38402 Saint-Martin-d'Hères cedex (France) Frederic.Mouton@ujf-grenoble.fr 